\title{
Study of Ferroelectric Phase Transition in Triglycine Selenate Crystal
}

\author{
Prabhat Chandra Khanduri ${ }^{1 *} \cdot$ Trilok Chandra Upadhyay $^{2}$ \\ ${ }^{1}$ Government Polytechnic College Pabau, Uttarakhand \\ ${ }^{2}$ Department of Physics, HNB Garhwal University, Srinagar (Garhwal) 246174, India
}

*Corresponding author Email: prabhatkhanduri@yahoo.com

Received: 29.8.2021; Revised: 8.11.2021; Accepted: 16.11.2021

(O) Society for Himalayan Action Research and Development

\begin{abstract}
Triglycine selenate (TGSe) is a Hydrogen bondend order disorder type ferroelectric, which go through a structural phase transition at $T_{c}=295 \mathrm{~K}$. TGSe is very similar to the Triglycine Sulphate crystal, which is extensively used in infrared detection. In the present paper we are using double time thermal Green's function method and modified pseudospin model to discuss ferroelectric behavior of TGSe crystal. Expressions for shift, width, soft mode frequency, dielectric constant, loss and transition temperature have been evaluated. By fitting model values of physical quantities in the theoretical expressions thermal dependence of soft mode frequency, dielectric constant and loss have been calculated in the vicinity of transition temperature. Theoretical results are in well agreement with experimental results of other researchers.
\end{abstract}

Keywords: TGSe, Ferroelectric, Dielectric Constant, Soft mode frequency, Tangent loss

\section{Introduction}

The Triglycine selenate $\left[\left(\mathrm{NH}_{2} \mathrm{CH}_{2} \mathrm{COOH}\right)_{3} \mathrm{H}_{2} \mathrm{SeO}_{4}\right.$, abridged as TGSe] belongs to a very interesting set of hydrogen bonded ferroelectrics and bears a close resemblance to vastly studied TGS family. TGSe crystals go through a archetypical order-disorder phase transition at about $\mathrm{T}_{\mathrm{c}} \sim 295 \mathrm{~K}$. This transition from paraelectric phase to ferroelectric phase is associated with transformation in space group symmetry from $\mathrm{P} 2{ }_{1} / \mathrm{m}$ to $\mathrm{P} 2_{1}$. Lattice parameters for TGSe are $\mathrm{a}=9.54 \AA \mathrm{A}, \mathrm{b}=12.92 \AA, \mathrm{c}=5.86 \AA$ and $\beta=110^{\circ}$.

TGSe crystals have similar space group symmetries as of TGS crystal [Kay and Kleinberg 1973]. There are various other similarities found by various researchers [Itoh and Mitsui 1973, Choudhury and Chitra et. al. 2004, Balasubramanian and Krishnan 1963] between these two crystals. In both crystals, molecular units from the viewpoint of the structural ferroelectric phase transition are $-\mathrm{NH}_{3}{ }^{+}$group connected with one of the three glycine ions and the hydrogen bond between the residual glycine ions [Balasubramanian and Krishnan 1963]. The most important discrepancy between the structure of TGSe and TGS is bearing of $\mathrm{SeO}_{4}{ }^{+}$group instead of $\mathrm{SO}_{4}{ }^{+}$group. As a result of this its specific heat peak is six times larger in comparison with TGS [Ema 1983,Noheda and Lifante et. al. 1993]. Some properties of these crystals depend on the specifications of their growing techniques [Sirota and Tsedrik 1971] but the crystal does not show enormous change due to this. The Phase transition in TGSe crystals are analyzed by various researchers using different methods such as high sensitivity calorimetric 
technique [Romero and Gallardo et. al. 2004, Romero and Gallardo et. al. 2006], linear birefringence method [Iglesias and Castillo 1998, Przeslawski 2000].

Theoretical studies regarding TGSe type ferroelectric have been carried out by Chaudhuri and Choudhury et. al (1988) by taking psuedospin lattice coupled mode model as a tool. In present paper we are modifying this model to produce better and convincing results. This paper include loss tangent graph and some new formulas which were not present in our earlier paper [Khanduri and Upadhyay 2017] .

Outline of our paper is as follows. First with the help of Green's function approach we obtain the expressions for shift, width, soft mode frequency, dielectric constant, tangent loss and transition temperature in section 2. In the succeeding section we explain and discuss about results and obtained formulas in section 2. Our paper is accomplished with a conclusion in section 4 .

\section{Calculation}

In present study to explain phase transition of TGSe crystal, the Hamiltonian used is

$$
\begin{gathered}
H=-2 \Omega \sum_{i}\left(S_{1 i}^{x}+S_{2 i}^{x}\right)-\sum_{i j}\left[J_{i j}\left(S_{1 i}^{z} S_{1 j}^{z}+S_{2 i}^{z} S_{2 j}^{z}\right)+K_{i j} S_{1 i}^{z} S_{2 j}^{z}\right]-\sum_{k} V_{i k}\left(S_{1 i}^{z} A_{k}+S_{2 j}^{z} A_{k}^{\dagger}\right)+\frac{1}{4} \sum_{k} \omega_{k}\left(A_{k}^{\dagger} A_{k}+B_{k}^{\dagger} B_{k}\right) \\
+\sum_{k_{1} k_{2} k_{3}} V^{(3)}\left(k_{1}, k_{2}, k_{3}\right) A_{k_{1}} A_{k_{2}} A_{k_{3}}+\sum_{k_{1} k_{2} k_{3} k_{4}} V^{(4)}\left(k_{1}, k_{2}, k_{3}, k_{4}\right) A_{k_{1}} A_{k_{2}} A_{k_{3}} A_{k_{4}}-\sum_{i k} V_{i k} S_{1 i}^{x} A_{k}-\sum_{i k} V_{i k} S_{2 i}^{x} A_{k}^{\dagger}
\end{gathered}
$$

where $\Omega$ is proton tunneling frequency, $\mathrm{J}$ and $\mathrm{K}$ are coupling constants corresponding to coupling in same lattice and different lattices respectively, $V_{i k}$ is spin-lattice interaction constant, $\omega_{k}$ is phonon frequency, $S^{z}$ and $S^{x}$ are components of pseudospin variable $\mathrm{S}, A_{k}$ and $B_{k}$ are operators corresponding to position and momenta, $V^{(3)}\left(k_{1}, k_{2}, k_{3}\right)$ are $V^{(4)}\left(k_{1}, k_{2}, k_{3}, k_{4}\right)$ third and fourth order atomic force constants.

This Hamiltonian is modified pseudospin lattice coupled mode model. For modifying model we are introducing here third and fourth order phonon anharmonic interaction and extra spin-lattice coupling terms. Introduction of anharmonic terms leads to explanation of dynamic characteristics of TGSe and extra spin-lattice coupling is an indirect coupling between tunneling motion of one proton and other proton, which modulates distance between two equilibrium sites in $\mathrm{O}-\mathrm{H} \cdot \cdot \mathrm{O}$ bonds.

We shall evaluate double time thermal Green's function given below

$$
\mathrm{G}_{\mathrm{ij}}\left(t-t^{\prime}\right)=\left\langle\left\langle S_{i}^{z}(t) ; S_{j}^{z}\left(t^{\prime}\right)\right\rangle\right\rangle=-i \theta\left(t-t^{\prime}\right)\left\langle\left[S_{i}^{z}(t), S_{j}^{z}\left(t^{\prime}\right)\right]\right\rangle
$$


where angular brackets denote the average over the grand canonical ensemble and $\theta\left(t-t^{\prime}\right)$ is the Heaviside's step function which is 1 for $\left(t-t^{\prime}\right)$ greater than 0 or 0 otherwise. We differentiate Green's function mentioned above twice with respect to times $t$ and $t^{\prime}$, then Fourier transforming and putting into Dyson's equation form

$$
\mathrm{G}_{\mathrm{ij}}(\omega)=\mathrm{G}_{\mathrm{ij}}^{0}(\omega)+\mathrm{G}_{\mathrm{ij}}^{0}(\omega) \tilde{\mathrm{P}}(\omega) \mathrm{G}_{\mathrm{ij}}^{0}(\omega)=G_{0}(\omega)+G_{0}(\omega) \tilde{\pi}(\omega) G_{0}(\omega)
$$

we get a Green function which contains higher order Green's function and simpler Green's function. Higher order Green's functions are evaluated by decoupling schemes while simple Green's functions by Zeroth order approximation. The Green's function which we at last obtain by this procedure is

$$
G_{i j}(\omega)=\frac{\Omega\left\langle S_{1 i}^{x}\right\rangle}{\pi\left[\omega^{2}-\hat{\Omega}^{2}-2 i \Omega \Gamma(\omega)\right]}
$$

where $\hat{\Omega}^{2}=\left(2 J\left\langle S_{1}^{z}\right\rangle+K\left\langle S_{2}^{Z}\right\rangle\right)^{2}+4 \Omega^{2}-4 J \Omega\left\langle S_{1}^{X}\right\rangle-2 \Omega K\left\langle S_{2}^{X}\right\rangle+\Delta(\omega)$

From Eq (2) it is clear that final Green's function contain Shift $\Delta(\omega)$ and width $\Gamma(\omega)$. Formula for these two is given in our previous paper [Khanduri P C and Upadhyay T C 2017].

Solving Eq (2) self consistently we obtain

$$
\hat{\Omega}^{2}=\frac{1}{2}\left(\tilde{\tilde{\omega}}_{k}^{2}+\tilde{\widetilde{\Omega}}^{2}\right) \pm \frac{1}{2}\left[\left(\tilde{\widetilde{\omega}}_{k}^{2}-\tilde{\widetilde{\Omega}}^{2}\right)^{2}+4\left\{\begin{array}{l}
4 \Omega^{2} V_{i k}^{2}\left\langle S_{1}^{x}\right\rangle \omega_{k}+\frac{8 \Omega a V_{i k}^{2}\left\langle S_{1}^{z}\right\rangle \omega_{k}}{b}+ \\
\frac{2 V_{i k}^{2} J_{i j}^{2}\left\langle S_{1}^{x}\right\rangle\left\langle S_{1}^{z}\right\rangle^{2} \omega_{k}+V_{i k}^{2} J_{i j}^{2}\left\langle S_{1}^{x}\right\rangle\left\langle S_{1}^{z}\right\rangle^{2} \omega_{k}}{\Omega} \\
+\frac{2 V_{i k}^{4}\left\langle S_{1}^{x}\right\rangle N_{k} \omega_{k}}{\Omega}+\frac{2 a V_{i k}^{4}\left\langle S_{1}^{z}\right\rangle N_{k} \omega_{k}}{b \Omega}
\end{array}\right]\right]^{1 / 2}
$$

In above equation the frequency corresponding to negative sign is soft mode frequency. From above equation it is clear that soft mode frequency depends explicitly on phonon frequency as well as spin lattice interaction terms.

The soft mode frequency approaches to zero, when the phase transition temperature is approached, which ultimately determine the limit of the paraelectric phase. The soft mode frequency can be expand in the power of $\left(T-T_{C}\right)$ around its value at $T_{C}$, when temperature reaches near transition temperature, which yields

$$
\hat{\Omega}_{-}^{2}=\frac{\Omega^{2} \tilde{J}(0)}{2 k_{B} T_{C}^{2}} \sec h^{2}\left(\frac{\Omega}{2 k_{B} T_{C}}\right)\left(T-T_{c}\right)=K\left(T-T_{C}\right)
$$

Where $\mathrm{k}_{\mathrm{B}}$ is the Boltzmann's constant $\mathrm{T}_{\mathrm{C}}$ is evaluated from Eq. (4) by using the lattice instability condition i.e. soft mode frequency approaches zero at the transition temperature, $\mathrm{T}_{\mathrm{C}}$. This condition gives 


$$
T_{c}=\frac{\eta}{2 k_{B} \tanh ^{-1}\left(\frac{\eta^{3}}{4 \Omega^{2} \widetilde{J}_{i j}}\right)},
$$

Where $\eta^{2}=\left(2 J_{0}-K_{0}\right)^{2} \sigma^{2}+4 \Omega^{2},\left\langle S_{1}^{Z}\right\rangle=-\left\langle S_{2}^{z}\right\rangle=\sigma$ and $\tilde{J}_{i j}=\left(2 J_{i j}+K_{i j}\right) \frac{2 \bar{V}_{i k}^{2} \omega_{k}^{2}}{\tilde{\widetilde{\omega}}_{k}^{2}}$

Equation (5) shows that the transition or Curie's temperature $\left(T_{c}\right)$ is explicit function of the tunneling factor and the third and fourth-order phonon anharmonic interactions via $\widetilde{J}_{i j}$ function.

Now from

$$
\varepsilon=1+4 \pi\left\{-\lim _{x \rightarrow 0} 2 \pi N \mu^{2} G_{i j}(\omega+i x)\right\}
$$

Dielectric Constant is obtained as

$$
\varepsilon=-\frac{8 \pi N \mu^{2} \Omega\left\langle S_{1}^{x}\right\rangle}{\left(\omega^{2}-\hat{\Omega}^{2}\right)}
$$

Ratio of imaginary to real parts of dielectric constant is loss of tangent, i.e.

$$
\tan \delta=\frac{\text { imaginary } \quad \varepsilon}{\operatorname{Re} a l \quad \varepsilon}=-\frac{2 \Omega \Gamma(\omega)}{\left(\omega^{2}-\hat{\Omega}^{2}\right)}
$$

\section{Results and Discussion}

By using the model values of a variety of physical quantities which appear in the evaluated expressions, thermal dependence of soft mode frequency, dielectric constant and loss tangent for different temperatures have been calculated and shown in figures 1,2 and 3. Model parameters for TGSe crystal used in present paper are given below.

\begin{tabular}{ccccccccccc}
$T_{c}$ & $C$ & $\Omega$ & $J$ & $K$ & $V_{i k}$ & $A_{k}$ & $N \times 10^{-21}$ & $\mu \times 10^{18}$ & $\omega_{k}$ \\
$(K)$ & $(k)$ & $\left(\mathrm{cm}^{-1}\right)$ & $\left(\mathrm{cm}^{-1}\right)$ & $\left(\mathrm{cm}^{-1}\right)$ & $\left(\mathrm{cm}^{-1}\right)$ & & $\left(\mathrm{cm}^{-3}\right)$ & esu & $\left(\mathrm{cm}^{-1}\right)$ \\
\hline 295.5 & 4727 & 0.2 & 320 & 160 & 0.01 & 10.2 & 3.81 & 3.43 & 6.2
\end{tabular}




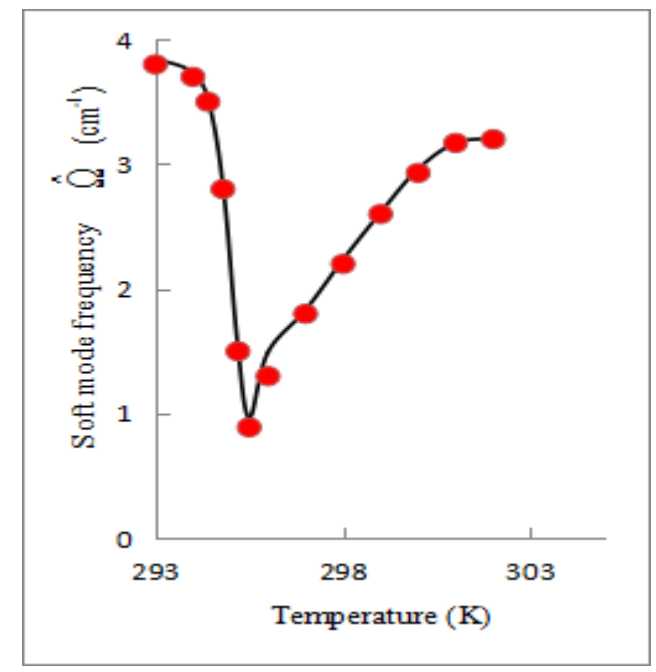

Fig 1. Thermal dependence of soft mode frequency of TGSe crystal (-our results, experimentally correlated vales for dielectric data)

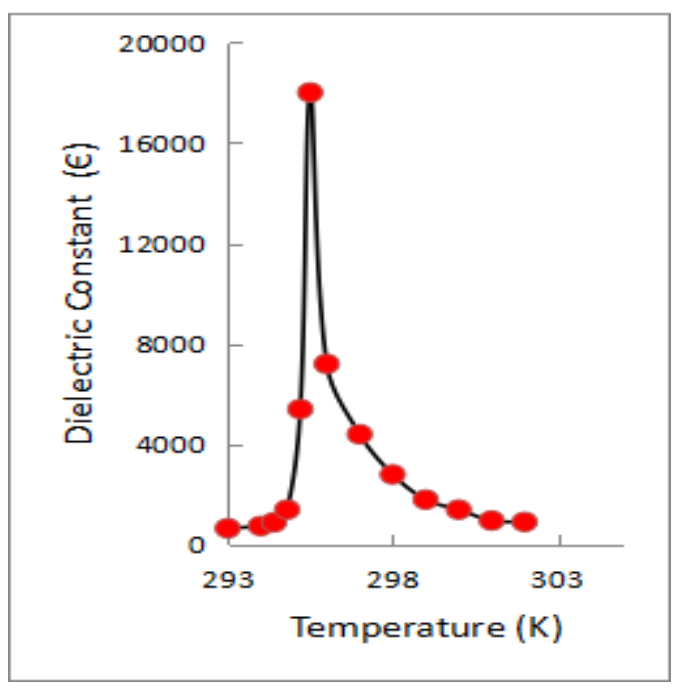

Fig 2. Thermal dependence of dielectric constant of TGSe crystal (-our results, experimentally values for dielectric data)

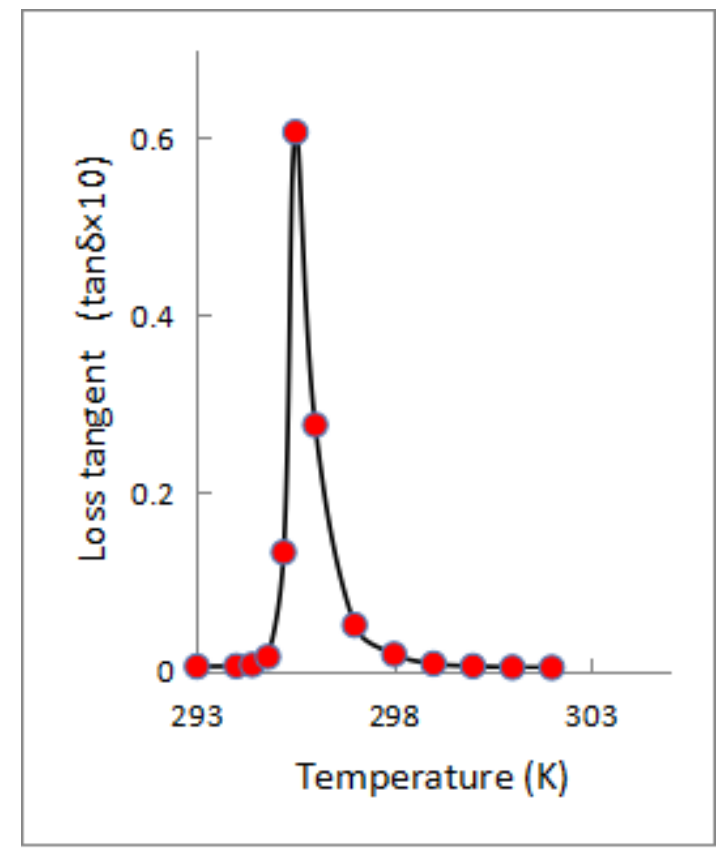

Fig 3. Thermal dependence of tangent loss of TGSe crystal (-our results $\bigcirc$ experimentally correlated vales for dielectric data)

From fig 1 which is the outcome of expression (3) evaluated in section 2, it is clear that soft mode frequency decreases as we approach from low temperature towards Curie temperature. At Curie temperature it becomes infinitesimally small and further increase in temperature leads to increase in soft mode frequency. 
Similarly from fig 2 (outcome of expression (7)) and fig 3 (outcome of expression (8)) it is clear that the dielectric constant and tangent loss first increases as temperature increases. They become enormously large at transition temperature, further increase in temperature leads to decrease in their values. Our findings agree with experimental results of Polandov and Mylov et. al. (1968).

Earlier researchers [Chaudhuri and Choudhury et. al. 1988] have not considered phonon anharmonic interactions and also some spin-lattice interactions. Correlations are decoupled by them in an early stage. Due to this some significant interactions vanished from their calculations. If we neglect these corrections our results reduces to the results of Chaudhuri and Choudhury et. al. (1988). Theses corrections give better understanding and more precise results.

\section{Conclusion}

It can be concluded from above discussion that modification of PLCM model by introducing third and fourth order phonon anharmonic interactions and extra spin-lattice coupling terms assist explanation of phase transition and dielectric behaviour of TGSe crystal. Theoretical results are in agreement with experimental results witnessed the applicability of the present modified model for TGSe crystal.

\section{References}

Balasubramanian K and Krishnan R S (1963). Raman spectrum of triglycine selenate. Proc. Ind. Acad. Sci: 209-215.

Chaudhuri B K, Choudhury K R and Banerjee S (1988). Green's-function theory of ferroelectric phase transition in hydrogen-bonded triglycine sulphate with the pseudospin-lattice coupled-mode mode: A unified theory of structural pase transitions. I. Static and dynamic properties. Physical Review (B) 38(1): 689 .

Choudhury R R, Chitra R and Ramanadham M (2004). Ferroelectric phase transition in triglycine selenate: an interpretation based on its structure and its comparison with triglycine sulphate. Phase transition 77(4): 385-395.

Ema K (1983). Critical behavior in the heat capacity of ferroelectric TGS, TGSe and TGFB. J. Phys Soc. Jpn $52: 2798$.

Iglesias T, Castillo J.R.F.D., Cereceda N, Lifante G, Przeslawski J and Gonzalo J A (1998), Temperature dependence of the order parameter at the quasi-tricritical phase transition in ferroelectric TGSe. Ferroelectrics, 208: 273.

Itoh K and Mitsui T (1973), Studies of the Crystal Structure of Triglycine Sulfate in Connection with its Ferroelectric Phase Transition. Ferroelectric 5 : 235-251.

Kay M I and Kleinberg R (1973), The crystal structure of triglycine sulphate. Ferroelectric $5: 45-52$.

Khanduri P C and Upadhyay T C (2017). Thermal variation of vibrational frequency and dielectric constant in triglycine selenate crystal. Journal of Pure Applied and Industrial Physics 7(2) : 29-34. 
Noheda B, Lifante G and Gonzalo J A (1993). Specific heat and Quadrupole interactions in uniaxial ferroelectrics. Ferroelectrics Letter Section 15 :109-114.

Polandov I.N., Mylov V.P., Strukov B.A. and Varikash V.M. (1968), Investigation of ferroelectric properties of trilycine selenate at high hydrostatic pressures. Soviet Physics-Solid State 10: 1092.

Przeslawski J (2000), Linear birefringence derivatives for TGSe and TGFB ferroelectric crystals.Ferroelectrics $237: 145-152$

Romero F J , Gallardo M C , Jimenez J and Cerro J D (2006), Influence of a weak dc electric field on tricritical phase transition in TGSe : Evidence of different specifi heat behaviour on cooling and heating runs. J. Phys. Codens. Matter, 18(44): 10075.

Romero F.J, Gallardo M.C. Jimenez J and Czarnecka A and Cerro J.D. (2004). Evidence of Landau tricritial behaviour in TGSe by calorimetric measurements: Effect of a weak uniaxial stress. J Phys Condens. Matter, 16(43): 7637.

Sirota N N, Tsedrik M S and Margolin L N (1971). Study of dielectric permeability and dielectric loss angle tangent to triglycine selenate depending on the conditions of ghrowing. Kristall und Technik 6(2): 240 . 